\title{
Conway's Question: The Chase for Completeness
}

\author{
Dikran Dikranjan · Elena Martín Peinador • \\ Vaja Tarieladze
}

\begin{abstract}
We study various degrees of completeness for a Tychonoff space $X$. One of them plays a central role, namely $X$ is called a Conway space if $X$ is sequentially closed in its Stone-Čech compactification $\beta X$ (a prominent example of Conway spaces is provided by Dieudonné complete spaces). The Conway spaces constitute a bireflective subcategory Conw of the category Tych of Tychonoff spaces. Replacing sequential closure by the general notion of a closure operator $C$, we introduce analogously the subcategory $\mathbf{C o n w}_{C}$ of $C$-Conway spaces, that turns out to be again a bireflective subcategory of Tych. We show that every bireflective subcategory of Tych can be presented in this way by building a Galois connection between bireflective subcategories of Tych and closure operators of Top finer than the Kuratowski closure. Other levels of completeness are considered for the (underlying topological spaces of) topological groups. A topological group $G$ is sequentially complete if it is sequentially closed in its Raikov completion $\tilde{G}$. The sequential completeness for
\end{abstract}

The paper was written while the author was visiting the Department of Topology and Geometry of Complutense University of Madrid and was supported by a grant of Grupo Santander (in the program Estancias de Doctores y Tecnólogos Extranjeros en la U.CM.). He takes the opportunity to thank his hosts for the warm hospitality and generous support.

The second and the third author acknowledge the financial aid received from MCYT, BFM2003-05878 and FEDER funds.

D. Dikranjan $(\varangle)$

Dipartimento di Matematica e Informatica, Università di Udine,

Via delle Scienze 206, 33100 Udine, Italy

e-mail: dikranja@dimi.uniud.it

E. Martín Peinador

Departamento de Geometría y Topología, Universidad Complutense de Madrid, Madrid, Spain e-mail: peinador@mat.ucm.es

V. Tarieladze

N. Muskhelishvili Institute of Computational Mathematics, Georgian Academy of Sciences, Akuri Str. 8, Tbilisi-0193, Georgia

e-mail: tar@gw.acnet.ge 
topological groups is stronger than Conway's property, although they coincide in some classes of topological groups, for example: free (Abelian) topological groups, pseudocompact groups, etc.

Keywords Dieudonné-complete space - Conway space •

Stone-Čech compactification • Compact abelian group • Pseudocompact group • Sequentially complete group $\cdot$ Functionally bounded set $\cdot$ Closure operator • Bireflective subcategory $\cdot$ Galois connection

Mathematics Subject Classifications (2000) Primary 22A05 - 22B05 - 54D25 • $54 \mathrm{H} 11 \cdot$ Secondary $54 \mathrm{~A} 35 \cdot 54 \mathrm{~B} 30 \cdot 54 \mathrm{D} 30 \cdot 54 \mathrm{H} 13$

\section{Introduction}

In the sequel we denote by Top the category of topological spaces and continuous maps and by Tych the full subcategory of Tychonoff spaces. For $A \subseteq X$ let $\operatorname{seq}(A)$ or $\operatorname{seq}_{X}(A)$ be the set of all those $x \in X$ for which there exists a convergent sequence extracted from $A$ with limit $x$. The set $A \subseteq X$ will be called sequentially closed in $X$ if $\operatorname{seq}(A)=A$ and it will be called sequentially dense in $X$ if $\operatorname{seq}(A)=X$ (so a subset $A \subseteq X$ is sequentially closed in $X$ iff no sequence in $A$ converges to a point of $X \backslash A)$.

\subsection{Two Properties of the Stone-Čech Compactification}

Let us start with two well-known properties of the Stone-Čech compactification $\beta X$ of a Tychonoff space $X$.

Fact 1.1 $\chi(y, \beta X)>\aleph_{0}$ for every $y \in \beta X \backslash X$.

Here we denote by $\chi(y, \beta X)$ the characater of $\beta X$ at the point $y \in \beta X$.

For normal spaces one can prove the stronger property - no sequence in $X$ converges to a point of $\beta X \backslash X$ :

Fact 1.2 If $X$ is normal, then $X$ is sequentially closed in $\beta X$.

These facts (whose proofs are given in Section 2) may leave the wrong impression that every Tychonoff space $X$ is sequentially closed in $\beta X$. Indeed, this assertion appeared in [6, Section V.6, Exercise 8]; in the second edition of the same book, Tychonoff is replaced by normal, so Exercise 8 in Section V.6 of [7] formulates Fact 1.2 together with the next question:

Question 1.3 If $X$ is not normal, is the statement of Fact 1.2 still true?

In $[26,13$, p. 244] Reid even 'proved' that every Tychonoff space $X$ is sequentially closed in $\beta X$. In Section 2.1 we offer a detailed analysis of that proof (see Remark 2.12 below). 
The next example showing that Fact 1.2 may fail for a non-normal Tychonoff space (and hence Question 1.3 has a negative answer) was suggested in Burckel's review MR 40 \#4685 to Flor's paper [16].

Example 1.4 Let $K:=\left(\omega_{1}+1\right) \times(\omega+1), p=\left(\omega_{1}, \omega\right)$ and let $T=K \backslash\{p\}$ be the Tychonoff plank. Then

(a) $K$ is compact, $T$ is a locally compact pseudocompact space and $K=\beta T$ ([17, $8.20]$ or $[34,14.6])$.

(b) $T$ is not sequentially closed in $\beta T=K$. (Indeed, $x_{n}=\left(\omega_{1}, n\right) \rightarrow p \notin T$.)

The interest of the property isolated by Conway and the frequent misunderstandings of it largely justify the explicit introduction of the following notion:

Definition 1.5 A Tychonoff space is a Conway space (or has the Conway property) whenever $X$ is sequentially closed in its Stone-Čech compactification $\beta X$.

According to Fact 1.2, all normal spaces are Conway. As we have noted, the Tychonoff plank presents an example of a non-Conway space.

An internal characterization of Conway spaces was obtained in [10], based on the notion of a $\mathcal{C}^{*}$-Cauchy sequence: namely, a sequence $\left(x_{n}\right)_{n \in \mathbb{N}}$ in a Tychonoff space $X$ such that the sequence $\left(f\left(x_{n}\right)\right)_{n \in \mathbb{N}}$ is convergent for every continuous function $f: X \rightarrow[0,1]$ (these are precisely the sequences $\left(x_{n}\right)_{n \in \mathbb{N}}$ in $X$ converging in $\beta X$ ). Although we are not going to use this characterization here, we give it for the sake of completeness.

Theorem 1.6 [10] For a Tychonoff space $X$ the following are equivalent:

(1) $X$ is a Conway space;

(2) X admits a compatible sequentially complete uniformity;

(3) every $\mathcal{C}^{*}$-Cauchy sequence $\left\{x_{n}: n \in \mathbb{N}\right\} \subset X$ converges in $X$;

(4) $X$ is sequentially closed in its Dieudonné completion $\mu X$.

Let us recall that a space $X \in \mathbf{T y c h}$ is Dieudonné-complete if $X$ admits a complete compatible uniformity. Equivalently, if $\mathrm{X}$ is complete in the fine uniformity. The Dieudonné completion of a Tychonoff space $X$, denoted by $\mu X$, is the completion with respect to the fine uniformity on $X$.

The above theorem gives clear evidence that Conway's property should be interpreted as a completeness property. However, it cannot be compared to Čechcompleteness since Čech-complete (and even locally compact) spaces need not be Conway (e.g., the Tychonoff plank). On the other hand, Conway spaces obviously need not be Čech-complete.

The class Conw of all Conway spaces determines a full subcategory of Tych which we shall still denote by Conw. Even if Conw is not closed under taking arbitrary subspaces (as every Tychonoff space is a subspace of a compact, hence Conway space), Conw is still quite well behaved:

Theorem 1.7 Conw is stable under taking arbitrary products and sequentially closed subspaces. 
This theorem can be proved by using the equivalence (1) $\Longleftrightarrow$ (2) of Theorem 1.6; however we shall obtain it also as a direct corollary of a much more general result (see Corollary 3.8 and Corollary 5.6). Observe that the nice behaviour of the Conway property with respect to products is in contrast with the failure of productivity for normality, countable compactness or countable paracompactness, each of them implying the Conway property (cf. Claim 2.11).

\subsection{Sequentially $\mathcal{P}$-closed Spaces}

Another motivation for this paper comes from the theory of $\mathcal{P}$-closed spaces. For a class $\mathcal{P}$ of topological spaces $X \in \mathcal{P}$ is called $\mathcal{P}$-closed if $X$ is closed in every space $Y \in \mathcal{P}$ containing $X$ as a subspace [3]. $\mathcal{P}$-closed spaces have been studied since the ground-breaking Memoir on compact spaces of Alexandroff and Urysohn, where $H$ closed spaces appeared for $\mathcal{P}=H$ the class of Hausdorff spaces. It is clear that the compact spaces are precisely the Tych-closed ones.

A modified approach to $\mathcal{P}$-closed spaces was adopted by Gotchev [18] (see also $[8,11])$. Call a $\mathcal{P}$-space sequentially $\mathcal{P}$-closed whenever $X$ is sequentially closed in any other $\mathcal{P}$-space where it can be embedded. The next example explains the advantages of this notion in certain cases when there are not sufficiently many $\mathcal{P}$-closed spaces.

\section{Example 1.8}

(a) Let US denote the full subcategory of Top having as objects all spaces where convergent sequences have unique limits. Then a US-space $X$ is US-closed iff it is finite [8, Theorem 2,7], whereas $X$ is sequentially US-closed iff $X$ is sequentially compact $[8$, Theorem 1,3$]$.

(b) For the full subcategory SUS of Top having as objects all spaces where convergent one-to-one sequences have unique adherent point ${ }^{1}$ the SUS-closed spaces are the finite unions of convergent sequences [8, Theorem 2.8], whereas the sequentially SUS-closed spaces are the countably compact SUS-spaces [8, Theorem 1.4].

It turned out that the counterpart of (b) remains true also in the nicer category Tych.

Theorem 1.9 ([18, Theorem 2.7]) $X \in$ Tych is sequentially Tych-closed iff $X$ is countably compact.

This theorem and item (b) of Example 1.8 show that in this context the countably compact spaces are the sequential counterpart of the compact spaces. Hence, countably compact spaces can be considered as a special instance of Conway spaces arising in the framework of sequentially Tych-closed spaces.

In contrast to [10] we concentrate in this paper on categorical aspects of Conway spaces, giving in the first sections the topological motivations that led us to Conw as well as a detailed exposition of Reid's proof enhancing mainly its positive features.

\footnotetext{
1.e., a point $x$ such that every neighbourhood of $x$ contains infinitely many members of the sequence.
} 
In Section 2 we discuss properties of $C^{*}$-embedded discrete subsets and their impact on Conway spaces. In Sections 3, 4, 5 we give a more general approach to the category Conw of Conway spaces, based on closure operators in the sense of [12]. In Section 3 we give the necessary background on closure operators in order to obtain an easy proof of the stability properties of Conw. In Section 4 (Definition 4.1) we define the category $\mathbf{C o n w}_{C}$ of $C$-Conway spaces depending on a closure operator $C$ that determines (in analogy with the sequential case) the level of completeness of $X$ (i.e., closedness of $X$ in $\beta X$ ) in terms of $C$. We prove that $\mathbf{C o n w}_{C}$ is a bireflective subcategory of Tych, moreover, every bireflective subcategory of Tych can be obtained in this way. We consider here also the stronger notion of absolutely $C$-closed spaces (see Definition 4.2) following the classical pattern of $\mathcal{P}$-closed spaces, that is replacing $\mathcal{P}$ with $\mathbf{T y c h}$ and "closed" by " $C$-closed" (so for the sequential closure operator $C=S e q$ the absolutely Seq-closed spaces are precisely the countably compact spaces, according to Theorem 1.9). We consider various cases when these two notions coincide (Example 4.3, Proposition 4.5 and Theorem 4.11) and we show that the coincidence problem can be resolved by the introduction of an appropriate modification $\widetilde{C}$ of $C$, so that the $C$-Conway spaces coincide with the absolutely $\widetilde{C}$-closed spaces (Corollary 4.16). In Section 5 we offer further generalizations of the Conway property that allow for a better understanding of $\mathbf{C o n w}_{C}$ (e.g., among others, we easily deduce in Corollary 5.6 that sequentially closed subspaces of Conway spaces are still Conway).

In Section 6.1 we give our third motivation for the introduction of the Conway spaces: the Conway property is the precise level of completeness of a topological space $X$ that characterizes the sequential completeness of the free topological group $F(X)$ of $X$. Finally, Section 6.2 contains some results on topological groups whose underlying spaces are Conway.

\section{The Category Conw of Conway Spaces}

Here we develop ideas of Reid [26] to obtain criteria for extendibility of appropriate continuous functions. As a by-product we obtain all necessary tools to prove Facts 1.1 and 1.2 .

A $C^{*}$-embedded (discrete) subset $A$ of a Tychonoff space $X$ need not be closed (take for example any infinite discrete space $A$ and $X=\beta A$ ). Now we prove that discrete $C^{*}$-embedded sets still have some closedness property:

Claim 2.1 If $D$ is a discrete $C^{*}$-embedded subspace of a Tychonoff space $Y$, then $D$ is sequentially closed in $Y$.

Proof Assume that $D$ is not sequentially closed in $Y$. Then there exists a one-to-one sequence $\left(y_{n}\right)$ in $Y$ such that $y_{n} \rightarrow y$ in $Y$, where $y \notin D$. For the set $A=\left\{y_{n}: n \in\right.$ $\mathbb{N}$ \} consider the function $f: A \rightarrow[0,1]$, with $f\left(y_{2 n}\right)=0$ and $f\left(y_{2 n-1}\right)=1$. Extend arbitrarily $f$ to a function $\widehat{f}: D \rightarrow[0,1]$. Since $D$ is discrete, $\widehat{f}$ is continuous. Obviously, it cannot be extended to a continuous function $D \cup\{y\} \rightarrow[0,1]$. So it cannot be extended to $Y$ either. Therefore $D$ is not $C^{*}$-embedded, and this contradicts the assumption. 
As an immediate corollary of Claim 2.1 one concludes that every discrete space is Conway.

For a better understanding of Reid's argument let us give the following definition, suggested by his rather natural construction (see Remark 2.12).

Definition 2.2 Let $X$ be a Tychonoff space. An $R$-quadruple on $X$ is defined as a quadruple $\mathfrak{T}=\left(\mathcal{O},\left(x_{n}\right),\left(r_{n}\right),\left(t_{n}\right)\right)$, where

1. $\mathcal{O}=\left(U_{n}\right)_{n \in \mathbb{N}}$ is a sequence of non-empty open subsets of $X$ with disjoint closures,

2. $\left(x_{n}\right)_{n \in \mathbb{N}}$ is a sequence in $X$ such that $x_{n} \in U_{n}$ for every $n \in \mathbb{N}$,

3. $\left(r_{n}\right)_{n \in \mathbb{N}}$ a sequence of real numbers,

4. $t_{n}: X \rightarrow[0,1], n=1,2, \ldots$ is a continuous function such that $t_{n}\left(X \backslash U_{n}\right)=\{0\}$ and $t_{n}\left(x_{n}\right)=1$ for every $n \in \mathbb{N}$.

Theorem 2.3 Let $\mathfrak{T}=\left(\mathcal{O},\left(x_{n}\right),\left(r_{n}\right),\left(t_{n}\right)\right)$ be an R-quadruple on a Tychonoff space $X$. Then $\mathfrak{T}$ gives rise to an open set $U=\bigcup_{n \in \mathbb{N}} U_{n}$ and a well-defined function $t=$ $\sum_{n \in \mathbb{N}} r_{n} t_{n}: X \rightarrow \mathbb{R}$ that obviously satisfies $t\left(x_{n}\right)=r_{n}$ and $t(x)=0$ for all $x \in X \backslash U$. Moreover, the following hold true:

(a) If $\mathcal{O}$ is locally finite or $r_{n} \rightarrow 0$, then $t$ is continuous.

(b) If $t$ is continuous, then for every $\varepsilon>0$ the family

$$
\mathcal{O}_{\varepsilon}=\left\{\left\{x \in U_{n}:|t(x)|>\varepsilon\right\}, n=1,2, \ldots\right\}
$$

is locally discrete.

(c) If $X$ is pseudocompact, then the continuity of timplies that $r_{n} \rightarrow 0$.

Proof

(a) Assume $\mathcal{O}$ is locally finite. Then $\bar{U}=\bigcup_{n} \bar{U}_{n}$ and $\mathcal{O}_{1}=\left\{\bar{U}_{n}\right\}_{n}$ is still a locally finite family of closed sets. Let $F=X \backslash U$. The family $\{F\} \cup \mathcal{O}_{1}$ provides a closed locally finite cover of $X$. The function $t$ is obviously continuous when restricted to each member of this family. Hence $t$ is continuous.

Now assume that $r_{n} \rightarrow 0$. As $\left|\sum_{k=n+1}^{\infty} r_{k} t_{k}(x)\right| \leq \sup _{k>n}\left|r_{k}\right|, n=1,2, \ldots$ for every $x \in X$, we have

$$
\sup _{x \in X}\left|\sum_{k=n+1}^{\infty} r_{k} t_{k}(x)\right| \leq \sup _{k>n}\left|r_{k}\right| \rightarrow 0, n \rightarrow \infty .
$$

Consequently,

$$
\sup _{x \in X}\left|\sum_{k=1}^{n} r_{k} t_{k}(x)-t(x)\right|=\sup _{x \in X}\left|\sum_{k=n+1}^{\infty} r_{k} t_{k}(x)\right| \leq \sup _{k>n}\left|r_{k}\right| \rightarrow 0, n \rightarrow \infty .
$$

Therefore the function $t$, as the uniform limit of the sequence $\left(\sum_{k=1}^{n} r_{k} t_{k}\right)_{n \in \mathbb{N}}$ of continuous functions, is continuous as well.

(b) Now assume that $t$ is continuous and pick an $\varepsilon>0$. Since the family $\mathcal{O}_{\varepsilon}$ refines $\mathcal{O}$, it is obviously locally discrete at the points of $U$ (because $\mathcal{O}$ has the same property). Now take any $x \in X \backslash U$. As $t(x)=0$, the continuity at $x$ yields $f(V) \subseteq(-\varepsilon, \varepsilon)$ for some neighbourhood $V$ of $x$. Obviously $V$ cannot meet any member of $\mathcal{O}_{\varepsilon}$. 
(c) Suppose $X$ is pseudocompact. Then for every $\varepsilon>0$ the family $\mathcal{O}_{\varepsilon} \backslash\{\emptyset\}$ must be finite (as a locally finite family of open non-empty sets of the pseudocompact space $X$ ). Thus $\left|r_{n}\right| \leq \varepsilon$ for all but finitely many $n$. Hence we can immediately conclude that $r_{n} \rightarrow 0$.

In order to better emphasize the strength of the condition of continuity of $t$ we isolate the following immediate corollary of Theorem 2.3.

Corollary 2.4 For an R-quadruple $\mathfrak{T}=\left(\mathcal{O},\left(x_{n}\right),\left(r_{n}\right),\left(t_{n}\right)\right)$ on a pseudocompact Tychonoff space $X$ the following two properties are equivalent:

(1) $r_{n} \rightarrow 0$

(2) $t$ is continuous.

Proof (1) implies (2) by Theorem 2.3 (a). Since $X$ is pseudocompact, (2) implies (1) by Theorem 2.3 (c).

Now we use Theorem 2.3 to produce countable closed, discrete $C^{*}$-embedded subsets.

Corollary 2.5 Let $\left(W_{n}\right)_{n \in \mathbb{N}}$ be a locally finite sequence of non-empty open subsets of a Tychonoff space $X$ and let $x_{n} \in W_{n}, n=1,2, \ldots$ Then the range $A \subset X$ of the sequence $\left(x_{n}\right)_{n \in \mathbb{N}}$ is a closed, discrete $C$-embedded (and hence, $C^{*}$-embedded) subset of $X$.

Proof Obviously, $A$ is closed and discrete. We can suppose that the set $A$ is infinite and coincides with the range of a one-to-one subsequence $\left(x_{k_{n}}\right)_{n \in \mathbb{N}}$ of $\left(x_{n}\right)_{n \in \mathbb{N}}$. Since the topological space $X$ is regular and $A$ is discrete, there exists a sequence $\mathcal{O}=$ $\left(U_{n}\right)_{n \in \mathbb{N}}$ of open sets with pairwise disjoint closures such that $x_{k_{n}} \in U_{n} \subset W_{k_{n}}, n=$ $1,2, \ldots$ Let now $f: A \rightarrow \mathbb{R}$ be any function and $r_{n}=f\left(x_{k_{n}}\right), n=1,2, \ldots$ For every $n \in \mathbb{N}$ define a continuous function $t_{n}: X \rightarrow[0,1]$, such that $t_{n}\left(x_{k_{n}}\right)=1$ and $t_{n}\left(X \backslash U_{n}\right)=\{0\}$. Let $t=\sum_{n \in \mathbb{N}} r_{n} t_{n}$ be the function associated with the $R$-quadruple $\mathfrak{T}=\left(\mathcal{O},\left(x_{n}\right),\left(r_{n}\right),\left(t_{n}\right)\right)$. Then $t$ is well defined and $t\left(x_{k_{n}}\right)=f\left(x_{k_{n}}\right)$ for every $n \in \mathbb{N}$. Hence, $t$ extends $f$. Moreover, since $\mathcal{O}$ is locally finite, by Theorem 2.3 (a) $t$ is continuous. Thus $A$ is $C$-embedded (it is clear that if $f$ is a bounded function, then $t$ is bounded as well).

Fact 1.1 immediately follows from the following stronger property:

Claim 2.6 If $Y$ is a Tychonoff space, $y \in Y$ and $\chi(y, Y)=\aleph_{0}$, then $X:=Y \backslash\{y\}$ is not $C^{*}$-embedded in $Y$.

Proof Let $\left(V_{n}\right)_{n \in \mathbb{N}}$ be a decreasing open local base at $y$ such that $W_{n}=V_{n} \backslash \bar{V}_{n+1} \neq$ $\emptyset, n=1,2, \ldots$. Note that the sequence $\left(W_{n}\right)_{n \in \mathbb{N}}$ of open sets is locally finite in $X$ as $\bigcap_{n} W_{n}=\emptyset$. Let $\left(x_{n}\right)_{n \in \mathbb{N}}$ be a sequence in $X$ such that $x_{n} \in W_{n}, n=1,2, \ldots$ Then by Corollary $2.5 A=\left\{x_{n}: n \in \mathbb{N}\right\} \subset X$ is a closed discrete $C^{*}$-embedded subset of $X$. Consider the function $f: A \rightarrow[0,1]$, with $f\left(x_{2 n}\right)=0$ and $f\left(x_{2 n-1}\right)=1$. Clearly, $f$ is continuous and since $A$ is $C^{*}$-embedded in $X$, the function $f$ has a continuous extension $\widehat{f}: X \rightarrow[0,1]$. On the other hand $x_{n} \rightarrow y$ but the sequence $\left(f\left(x_{n}\right)\right)$ has 
two cluster points, therefore the function $\widehat{f}$ does not admit a continuous extension to $Y$. Hence $X$ is not $C^{*}$-embedded in $Y$.

Corollary 2.7 Let X be a countably paracompact Tychonoff space. Then every countable closed discrete subset $A$ of $X$ is $C$-embedded in $X$.

Proof Write $A=\left\{x_{1}, x_{2}, \ldots\right\}$ and $U_{0}=X \backslash A$. Since $A$ is a closed discrete subspace of $X$, we can find again a sequence $\left(U_{n}\right)_{n \in \mathbb{N}}$ of pairwise disjoint open subsets of $X$ with

$$
U_{n} \ni x_{n}, n=1,2, \ldots
$$

Then $\left\{U_{0}, U_{1}, U_{2}, \ldots\right\}$ is an open cover of $X$. Since $X$ is countably paracompact, there exists a locally finite open cover $\left\{W_{0}, W_{1}, W_{2}, \ldots\right\}$ of $X$ with

$$
W_{i} \subset U_{i}, i=0,1,2, \ldots
$$

(see [15, Theorem 5.2.1, p. 316]). Since $\left(U_{n}\right)_{n \in \mathbb{N}}$ is a pairwise disjoint sequence, from Eqs. 1 and 2 we have $x_{n} \in W_{n}, n=1,2, \ldots$ Now we can conclude with Corollary 2.5 that $A$ is $C$-embedded in $X$.

The above corollary suggests to introduce the following condition for a space $X$ :

Definition 2.8 A space $X$ is said to have the property (CCD) if the countable closed discrete subsets of $X$ are $C^{*}$-embedded in $X$.

Example 2.9 Here are examples of spaces having the property (CCD):

(a) Countably paracompact spaces satisfy (CCD) by Corollary 2.7.

(b) Normal spaces satisfy (CCD) as every closed subspace of a normal space is $C^{*}$ embedded.

(c) Finally, countably compact spaces satisfy (CCD) in a vacuous way.

Remark 2.10 In general, an arbitrary Tychonoff space $X$ need not satisfy (CCD). As Gillman-Jerison [17] suggest many authors have fallen into the trap of assuming the opposite (see [17, 8.21, p. 125]). Claim 2.11 shows, in particular, that each non-Conway space contains a countable, closed and discrete subset which is not $C^{*}$-embedded. On the other hand, (CCD) does not characterize the countably paracompact spaces, since there exists a normal Hausdorff space, which is not countably paracompact (M. E. Rudin [27]).

The next statement reveals the importance of (CCD) and covers Fact 1.2. 
Claim 2.11 A Tychonoff space $X$ satisfying (CCD) is a Conway space. In particular, normal spaces, as well as countably paracompact or countably compact spaces are Conway spaces.

Proof According to Example 2.9 it is sufficient to show that a space with (CCD) must be a Conway space.

Let $\left(x_{n}\right)$ be a sequence of distinct points from $X$ such that $x_{n} \rightarrow y \in \beta X \backslash X$, then $A=\left\{x_{n}: n \in \mathbb{N}\right\} \subset X$ is discrete and closed in $X$. Consider the function $f: A \rightarrow$ $[0,1]$, with $f\left(x_{2 n}\right)=0$ and $f\left(x_{2 n-1}\right)=1$. Since $A$ is discrete, $f$ is continuous. By the assumption (CCD), the countable closed discrete set $A$ is $C^{*}$-embedded in $X$, and consequently in $\beta X$. Let $\widehat{f}: \beta X \rightarrow[0,1]$ be a continuous extension of $f$. As $x_{n} \rightarrow$ $y \in \beta X$, then $\widehat{f}\left(x_{n}\right) \rightarrow \widehat{f}(y)$ and so, $f\left(x_{n}\right) \rightarrow \widehat{f}(y)$, which is a contradiction as $\left(f\left(x_{n}\right)\right)$ is not a convergent sequence.

Remark 2.12 Note that the condition (CCD) was substantially used in the above proof. Without any additional assumption about $X$, Reid argued as follows: let $A=$ $\left\{x_{n}: n \in \mathbb{N}\right\} \subset X$ be as in the proof of Claim 2.11. Then since $A$ is closed and discrete in $X$, there are open sets $U_{n} \ni x_{n}$ with pairwise disjoint closures and functions $t_{n}: X \rightarrow[0,1]$, such that $t_{n}\left(x_{n}\right)=1$ and $t_{n}\left(X \backslash U_{n}\right)=\{0\}$. Write $t=\sum_{n=1}^{\infty}(-1)^{n} t_{n}$ (i.e., in terms of Definition 2.2, $\left(\left(U_{n}\right),\left(x_{n}\right),\left((-1)^{n}\right),\left(t_{n}\right)\right)$ is an $R$-quadruple and $t$ the corresponding function). Claiming that $t$ is continuous he got a contradiction. As proved in Corollary 2.4, $t$ is not continuous if $X$ is pseudocompact and non-Conway (e.g., when $X=T$ is the Tychonoff plank).

Remark 2.13 Let us briefly comment on Claim 2.11.

(a) The fact that normal spaces are Conway spaces is apparently well-known; its validity was pointed out already in the above-mentioned review by Burckel (see also [34, Theorem 8.3.2, p.148], [25, Proposition IX.4.12]).

(b) That countably paracompact spaces are Conway spaces is seemingly proved in [24] (the authors had no access to this paper).

(c) The implications in (a) and (b) cannot be inverted (according to Theorem 1.7 the Conway property is productive while neither normality nor countable paracompactness are productive).

(d) In contrast to (c) we have no examples of a Conway space that does not satisfy condition (CCD).

\section{Background on Closure Operators}

\subsection{Birefletive Subcategories of Tych}

From now on we shall concentrate mainly on the subcategory Tych of Top.

Definition 3.1 A full subcategory $\mathbf{A}$ of Tych is called bireflective, if for every $X \in$ Tych there exists a continuous injection $\iota_{X}: X \rightarrow \rho X$ with dense image in $\rho X \in \mathbf{A}$, and such that for every continuous map $f: X \rightarrow A \in \mathbf{A}$ there exists a (necessarily unique) map $f_{1}: \rho X \rightarrow A$ with $f=f_{1} \circ \iota_{X}$. 
Standard examples of bireflective subcategories of Tych are the category Comp of compact spaces (with bireflection $j_{X}: X \rightarrow \beta X$ ) and the category Dieu of Dieudonné complete spaces. Let us note that Comp is the smallest bireflective subcategory of Tych (as $\iota_{X}: X \rightarrow \rho X$ must be a homeomorphism for every compact space $X$ and bireflective subcategories are closed under taking homeomorphic images). Moreover, for every bireflective subcategory of Tych the reflection map $\iota_{X}: X \rightarrow \rho X$ is an embedding with $X \subseteq \rho X \subseteq \beta X$ and for all $X \subseteq Y \subseteq \rho X$ one has $\rho Y=\rho X$.

The fact that every bireflective subcategory of Tych is closed under taking closed subspaces and arbitrary products is well known in the framework of category theory under a much more general setting [1]. Conversely, every full subcategory of Tych stable under taking closed subspaces and arbitrary products and containing the closed interval $[0,1]$ (or, equivalently, Comp) is bireflective.

The bireflective subcategory Dieu of Tych is generated by all metric spaces. The reflection $\mu X$ of $X$ is the completion with respect to the fine uniformity of $X$. Therefore, $X \subseteq \mu X \subseteq \beta X$. In particular, $\mu X=\beta X$ if and only if $X$ is pseudocompact. One can mention here also the category RComp of realcompact spaces sitting between Comp and Dieu. It is generated by the reals $\mathbb{R}$ (so the realcompact spaces are precisely the closed subspaces of the powers of $\mathbb{R}$ ) and its reflector $X \mapsto v X$ is known also as Hewitt compactification and satisfies $\mu X \subseteq v X \subseteq \beta X$.

\subsection{Closure Operators}

First we recall the following definition $[12,13]$.

A closure operator $C$ in the category Top of topological spaces is given by a family of maps $c_{X}: 2^{X} \longrightarrow 2^{X}, X \in \mathbf{T o p}$, such that for every $X$ in Top

- $\quad c_{X}$ is expansive : $M \subseteq c_{X}(M)$ for all $M \in 2^{X}$,

- $\quad c_{X}$ is monotone : $c_{X}(M) \subseteq c_{X}(N)$ for all $M \subseteq N \subseteq X$,

and for every continuous map $f: X \rightarrow Y$ in Top

- $\quad f\left(c_{X}(M)\right) \subseteq c_{Y}(f(M))$ for all $M \subseteq X$

A subset $M \subseteq X$ is $C$-closed $\left(C\right.$-dense) if $c_{X}(M)=M$ (resp., $\left.c_{X}(M)=X\right)$.

A closure operator $C$ of Top is:

- $\quad$ idempotent if $c_{X}(M)$ is $C$-closed for all $M \subseteq X, X \in \mathbf{T o p}$;

- weakly hereditary if $M$ is $C$-dense in $c_{X}(M)$ for all $M \subseteq X, X \in \mathbf{T o p}$;

- hereditary if $c_{Y}(M)=c_{X}(M) \cap Y$ for all $M \subseteq X, X \in$ Top and $M \subseteq Y \subseteq X$;

- additive if $c_{X}(M \cup N)=c_{X}(M) \cup c_{X}(N)$ for all $M, N \subseteq X, X \in$ Top;

- (finitely) productive, if $c_{X}\left(\prod_{i} M_{i}\right)=\prod_{i} c_{X_{i}}\left(M_{i}\right)$, whenever $X=\prod_{i \in I} X_{i}$ and $M_{i} \subseteq X_{i}$ for each $i \in I$ (when $I$ finite).

We say that a closure operator $C$ is finer than a closure operator $C^{\prime}$ (denoted by $\left.C \leq C^{\prime}\right)$ if $c_{X}(M) \subseteq c_{X}^{\prime}(M)$ holds for every space $X$ and every subspace $M$ of $X$. The closure operators form a large lattice in the sense that the meet $\bigwedge_{i \in I} C_{i}$ and the join 
$\bigvee_{i \in I} C_{i}$ of any family (possibly a proper class) $\left\{C_{i}: i \in I\right\}$ of closure operators exists, defined by

$$
\left(\bigwedge_{i \in I} c_{i}\right)_{X}(M)=\bigcap_{i \in I}\left(c_{i}\right)_{X}(M) \text { and }\left(\bigvee_{i \in I} c_{i}\right)_{X}(M)=\bigcup_{i \in I}\left(c_{i}\right)_{X}(M) .
$$

For closure operators $C$ and $C^{\prime}$ define the composition $C^{\prime} C$ and the co-composition $C^{\prime} * C$ by

$$
\left(c^{\prime} c\right)_{X}(M)=c_{X}^{\prime}\left(c_{X}(M)\right) \text { and }\left(c^{\prime} * c\right)_{X}(M)=c_{c_{X}(M)}^{\prime}(M) .
$$

For every closure operator $C$ there exists a finest idempotent closure operator $C^{\infty}$ with $C \leq C^{\infty}$, called the idempotent hull of $C$. To compute the idempotent hull $C^{\infty}$ one defines first the transfinite iterations of $C$ as follows: $C^{1}=C, C^{\alpha+1}=C C^{\alpha}$ for all ordinals $\alpha$ and $C^{\alpha}=\bigvee_{\beta<\alpha} C^{\beta}$ for limit ordinals $\alpha$. Then $C^{\infty}=\bigvee_{\alpha} C^{\alpha}$. (One can compute $c_{X}^{\infty}(M)$ also "from above" by taking the intersection of all $C$-closed subspaces of $X$ containing M.) More details can be found in [12] (in the general setting of abstract categories) and in [13] (in Top).

Example 3.2 The usual Kuratowski closure $K=\left(k_{X}\right)$ defined by $k_{X}(M)=\bar{M}$ is, of course, the inspiring example. Here are some more examples:

(a) the discrete closure operator $D$ defined by $d_{X}(M)=M$;

(b) the sequential closure Seq, defined by $\operatorname{Seq}=\left(\operatorname{seq} q_{X}\right)$

(c) the $\omega$-closure $\Omega=\left(\omega_{X}\right)$ defined by $\omega_{X}(M)=\bigcup\{\bar{A}: A \subseteq M,|A| \leq \omega\}$.

(d) the $\lambda$-closure $\Lambda=\left(\lambda_{X}\right)$, where $\lambda$ is an infinite cardinal, defined by

$$
\lambda_{X}(M)=\bigcup\{\bar{A}: A \subseteq M,|A| \leq \lambda\} .
$$

(e) the $\mathrm{k}$-closure $\mathrm{K}=\left(\mathrm{k}_{X}\right)$ defined by $\mathrm{k}_{X}(M)=\bigcup\{\overline{M \cap K}: K \subseteq X$, compact $\}$;

(f) the $G_{\delta}$-closure $G_{\delta}=\left(\left(g_{\delta}\right)_{X}\right)$ defined by

$$
\left(g_{\delta}\right)_{X}(M)=\left\{x \in X:\left(\forall G_{\delta} \text {-set } O \subseteq X\right) O \ni x \Rightarrow O \cap M \neq \emptyset\right\} ;
$$

(g) [14] the $f b$-closure defined, for $M \subseteq X$, by

$$
f b_{X}(M)=\bigcup\{\bar{B}: B \subseteq M, \text { functionally bounded }\} .
$$

All seven closure operators are weakly hereditary ${ }^{2}$, additive and productive, and all of them except $\mathrm{Seq}$ and $\mathrm{K}$ are also idempotent. This gives rise to two more closure operators, namely the idempotent hulls $S e q^{\infty}$ and $K^{\infty}$. Let us recall that $S e q^{\infty}$ is obtained by transfinite $\omega_{1}$ iterations of $S e q$, whereas no such common upper bound for all spaces exists for the number of iterations of $K$ in order to get $K^{\infty}$ [23]. Furthermore, all these closure operators are finer than the Kuratowski closure $K$, i.e., the $C$-closure of any subset $M$ is always contained in the usual closure of $M$. Therefore, in all seven cases, $C$-dense implies also dense in the usual sense (whereas $C$-closed subsets need not be closed).

${ }^{2}$ all of them except $\mathrm{K}$ and $f b$ are actually hereditary. 
One can describe many well-known properties of the topological space in terms of these closure operators as follows.

(a) $X$ is discrete precisely when $k_{X}=d_{X}$.

(b) $X$ is Fréchet-Urysohn precisely when $k_{X}=\operatorname{seq} q_{X}$, while $X$ has no non-trivial convergent sequences precisely when $\operatorname{seq} q_{X}=d_{X}$. Moreover, the space $X$ is sequential precisely when $k_{X}=\operatorname{seq} q_{X}^{\infty}$.

(c) $X$ has countable tightness precisely when $k_{X}=\omega_{X}$, while $\omega_{X}=d_{X}$ when the topology of $X$ is finer than the co-countable topology of $X$ (having as proper closed subsets all countable subsets of $X$ ).

(d) $X$ has tightness $\leq \lambda$ precisely when $k_{X}=\lambda_{X}$, while $\lambda_{X}=d_{X}$ when the topology of $X$ is finer than the co- $\lambda$ topology of $X$ (having as proper closed subsets all subsets of size $\leq \lambda$ of $X$ ).

(e) $X$ is a k-space precisely when $k_{X}=\mathrm{k}_{X}^{\infty}$, while $X$ has no infinite compact sets precisely when $\mathrm{k}_{X}=d_{X}$.

(f) If $X$ is a $P$-space then $k_{X}=\left(g_{\delta}\right)_{X}$, while $X$ has countable pseudo-character precisely when $\left(g_{\delta}\right)_{X}=d_{X}$.

The proof of the next lemma can be found in [12].

Lemma 3.3 If $C$ is a finitely productive closure operator of Top, and $H$ is a subgroup of a topological group $G$, then also $c_{G}(H)$ is a subgroup of $G$.

The next lemma follows directly from the definitions.

\section{Lemma 3.4 Let $C$ be a closure operator of Top.}

(a) If $f: X \rightarrow Y$ is a continuous surjective map and $M \subseteq X$ is $C$-dense, then $f(M)$ is $C$-dense in $Y$.

(b) If $C$ is (finitely) productive, $\left\{X_{i}: i \in I\right\}$ is a (finite) family of spaces and $M_{i} \subseteq X_{i}$ is $C$-dense for every $i \in I$, then $\prod_{i} M_{i}$ is $C$-dense in $\prod_{i} X_{i}$.

One can define closure operators of Tych in a similar way. Clearly, every closure operator of Top gives, by restriction, a closure operator of Tych (and every closure operator of Tych gives rise to a closure operator of Top, but we are not going to use it here, see [12]). All closure operators of Tych that will appear in the sequel will be finer than $K$ and satisfy $c_{X}(M)=c_{k_{X}(M)}(M)$ for every pair $M \subseteq X \in$ Tych (we shall briefly denote this by $C \leq_{h} K$ ). This will allow us to define the closure operator only for pairs with $M$ dense in $X$. Obviously, $C \leq_{h} K$ holds for all hereditary closure operators finer than $K$, as well as for $\mathrm{K}$ and $f b$. An example of a closure operator $C$

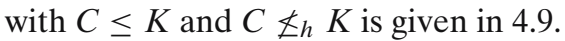

For the study of Conw we need also the following new properties of closure operators:

Definition 3.5 Let $C$ be a closure operator of Tych. We say that

(a) $C$ is $\beta$-hereditary if for every $X \in$ Tych and every dense subset $M$ of $X$ one has $c_{X}(M)=c_{\beta X}(M) \cap X$.

(b) stable, if for every $X \in \mathbf{C o m p}$ and every dense subset $M$ of $X$ one has $c_{X}(M)=$ $f\left(c_{\beta M}(M)\right)$, with $f=\beta i: \beta M \rightarrow X$, where $i$ is the inclusion $M \hookrightarrow X$. 
In fact, (a) is a weaker notion of hereditariness, while (b) says (in the standard terminology of closure operators) that $f$ is $C$-preserving.

\section{Example 3.6}

(a) $D, K, \mathrm{~K}$ and $f b$ are stable.

(b) For every infinite cardinal $\lambda$ the closure operator $\Lambda$ is stable.

(c) Seq is not stable (take the subspace $\mathbb{N}$ of the one-point Alexandorff compactification of $\mathbb{N}$ ). We shall see later that no power $S e q^{\alpha}$ of $S e q$ is stable (see Remark 4.7).

(d) The closure operators $\mathrm{K}$ and $f b$ are not $\beta$-hereditary.

Arbitrary meets of $\beta$-hereditary closure operators are $\beta$-hereditary, hence every closure operator $C$ of Tych admits a $\beta$-hereditary hull $\beta(C)$, namely a finest $\beta$ hereditary closure operator of Tych with $C \leq \beta(C)$. One can define explicitly $\beta(C)$ by setting $\beta(c)_{X}(M)=c_{\beta X}(M) \cap X$ for any dense subset $M$ of $X \in$ Tych. Clearly, $\beta(c)_{X}=c_{X}$ for every $X \in \mathbf{C o m p}$; moreover $C$ is $\beta$-hereditary if and only if $C=\beta(C)$. It is easy to see that $\beta(C)$ is idempotent whenever $C$ is idempotent, moreover $\beta(C)$ is stable if and only if $C$ is stable.

\subsection{The Conway Reflection}

The Conway reflection Con $X$ of a space $X$ is defined by $\operatorname{Con} X=\operatorname{seq}_{\beta X}^{\infty}(X)$; i.e., Con $X$ is the smallest sequentially closed subspace of $\beta X$ containing $X$. The term is justified by the next theorem.

Theorem 3.7 Conw is a bireflective subcategory of Tych containing Dieu. The reflector Tych $\rightarrow$ Conw is given by $X \mapsto$ Con $X$.

Proof Clearly, $X$ is dense in Con $X$. To verify the universal property take an arbitrary continuous map $f: X \rightarrow Y$, where $Y$ is a Conway space. Then $Y$ is sequentially closed in $\beta Y$. By the universal properties of $\beta X$ there exists a continuous map $l=\beta f: \beta X \rightarrow \beta Y$ such that $l \circ j_{X}$ (i.e., the restriction of $l$ to $X$ ) coincides with the composition $j_{Y} \circ f$. Since $s e q^{\infty}$ is a closure operator, $l(\operatorname{Con} X)=l\left(\operatorname{seq}_{\beta X}^{\infty}(X)\right) \subseteq$ $\operatorname{seq}_{\beta Y}^{\infty}(Y)=Y$. The restriction of $l$ to Con $X$ is the desired arrow $f_{1}:$ Con $X \rightarrow Y$. The uniqueness of $f_{1}$ follows immediately from the density of $X$ in $\operatorname{Con} X$.

Corollary 3.8 Conw is closed under taking closed subspaces and arbitrary products.

Proof This follows immediately from Theorem 3.7 and the well known fact that every bireflective subcategory of Tych is closed under taking closed subspaces and arbitrary products.

As far as stability under taking closed subspaces is concerned, we shall prove below a much stronger result (see Theorem 5.5).

From Corollary 3.8 we obtain an easy example of a non-normal Conway space that is not countably compact, namely $\mathbb{Z}^{\omega_{1}} \in$ Dieu. 


\section{The Galois Correspondence of Conway's Property}

\subsection{The Conway Property w.r.t. a Closure Operator}

One can define a counterpart $\mathbf{C o n w}_{C}$ of Conw for every closure operator $C$ finer than $K$ as follows.

Definition 4.1 Call a Tychonoff space $X C$-Conway if $X$ is $C$-closed in $\beta X$ and denote by Conw $_{C}$ the category of $C$-Conway spaces.

Clearly, for $C=\operatorname{seq}$ or $C=s e q^{\infty}$ we get the usual Conway spaces. Using a slight modification of the proof of Theorem 3.7 one can prove that the category $\mathbf{C o n w}_{C}$ of $C$-Conway spaces is a bireflective subcategory of $\mathbf{T y c h}$ (we shall give below the proof of a more general property, see Theorem 5.2). Consequently, $\mathbf{C o n w}_{C}$ is stable under taking closed subspaces and arbitrary products. Actually, one can prove a stronger property than just stability under taking closed subspaces: the category Conw $_{C}$ is stable under taking $C$-closed subspaces (note that closed implies $C$-closed as $C$ is finer than $K$ ). We shall prove a more general property below (see Theorem 5.5).

The notion of a $C$-Conway space should be compared with the following stronger one:

Definition 4.2 Call a Tychonoff space $X$ absolutely $C$-closed if $X$ is $C$-closed in every Tychonoff space $Y$ where it can be embedded.

Obviously, compact spaces are absolutely $C$-closed, while absolutely $C$-closed spaces are always $C$-Conway spaces, i.e.,

$$
\text { compact } \Longrightarrow C \text {-Conway } \stackrel{(\dagger)}{\Longrightarrow} \text { absolutely } C \text {-closed. }
$$

According to Remark 2.13, the implication $(\dagger)$ cannot be inverted for the sequential closure operator $s e q$, as absolutely Seq-closed spaces are the countably compact ones (by Theorem 1.9). Motivated by this example we provide a large class of closure operators for which the implication $(\dagger)$ in Eq. 3 can be inverted and ask in Question 7.5 to determine all these closure operators.

Contrary to $C_{\text {Conw }}$ the smaller subcategory of absolutely $C$-closed is not well behaved. It need not even be stable under finite products (take $C=S e q$, here finite products of countably compact spaces need not be countably compact). This justifies our initial interest in the larger subcategory Conw $\mathbf{C}_{C}$. We shall prove in the sequel that every full subcategory of Tych containing Comp and stable under products and taking closed subspaces has necessarily the form $\mathbf{C o n w}_{C}$. Moreover, $\mathbf{C o n w}_{C}=\mathbf{C o n w}_{C^{\prime}}$ for another closure operator $C^{\prime}$ such that every $C^{\prime}$-Conway space is also absolutely $C^{\prime}$-closed (see Theorem 4.11). In other words, the closure operator $C^{\prime}$ resolves the problem of the inversion of the implication $(\dagger)$ in Eq. 3 leaving the category Conw $\mathbf{w}_{C}=$ Conw $_{C^{\prime}}$ unchanged.

If $S e q \leq C$ for a closure operator $C$, then every $C$-Conway space is Conway. More generally, $C_{1} \leq C_{2}$ yields Conw $_{C_{1}} \supseteq$ Conw $_{C_{2}}$ (see Proposition 5.3). However, the equality $\mathbf{C o n w}_{C_{1}}=\mathbf{C o n w}_{C_{2}}$ may occur with distinct $C_{1}, C_{2}$ (for example, always $\left.\operatorname{Conw}_{C}=\operatorname{Conw}_{C}\right)$. 
In the next example we see that under certain circumstances $C$-Conway spaces are actually compact.

Example 4.3 Let us compare now the Conway property with compactness:

(a) One can immediately see that for $C=f b$ and $\mathrm{k}$, for a space $X \in$ Tych the following are equivalent:

(a $\left.\mathrm{a}_{1}\right)$ is $C$-Conway;

$\left(\mathrm{a}_{2}\right) \quad X$ is absolutely $C$-closed;

(a) $X$ is compact.

(b) Analogously, a countable space is $\Omega$-Conway iff it is compact.

In the next proposition we give a more general form of item (b). Actually, it gives a nice internal description of the $\Omega$-Conway spaces. Moreover, it shows that $\Omega$-Conway spaces are absolutely $\Omega$-closed. Recall that a space $X$ is $\omega$-bounded when every countable subset of $X$ is contained in a compact subset of $X$. Obviously, every $\omega$-bounded space is countably compact. In the next two proposition we make substantial use of the closure operators $\Omega$ and $\Lambda$ defined in Example 3.2.

Proposition 4.4 For a Tychonoff space $X$ the following are equivalent:

(a) $X$ is $\Omega$-Conway;

(b) $X$ is $\omega$-bounded;

(c) $X$ is an absolutely $\Omega$-closed space.

We shall give the proof in the following more general form. For every infinite cardinal $\lambda$ call a space $X$ is $\lambda$-bounded when every subset of $X$ of size $\leq \lambda$ is contained in a compact subset of $X$.

Proposition 4.5 For a Tychonoff space $X$ the following are equivalent:

(a) $X$ is $\Lambda$-Conway;

(b) $X$ is $\lambda$-bounded;

(c) $X$ is an absolutely $\Lambda$-closed space.

Proof

(a) $\rightarrow$ (b). If $X$ is $\Lambda$-Conway, then $X$ must be $\Lambda$-closed in $\beta X$. So for every countable subset $A$ of $X$ the closure $B$ (computed in $\beta X$ ) of $A$ must be contained in $X$. Since $B$ is compact, this proves that $X$ is $\lambda$-bounded.

(b) $\rightarrow$ (c). Every $\lambda$-bounded space is $\Lambda$-closed in any Hausdorff space, hence is absolutely $\Lambda$-closed.

$(\mathrm{c}) \rightarrow$ (a) is trivial.

The above proposition gives a proper class of distinct categories of $C$-Conway spaces, namely the categories $\mathbf{B}_{\lambda}$ of $\lambda$-bounded spaces. Note that for $\lambda<\mu$ one has $\mathbf{B}_{\lambda} \supset \mathbf{B}_{\mu}$ and the intersection of all $\mathbf{B}_{\lambda}$ 's is precisely Comp.

The next proposition shows that in Proposition 4.5 the equivalence of (a) and (c) for the closure operator $\Lambda$ can be generalized to all stable closure operators. 
Proposition 4.6 Let $C$ be a closure operator of $\mathbf{T y c h}$.

(a) If $C$ is stable, then every $X \in \mathbf{C o n w}_{C}$ is absolutely $C$-closed.

(b) If every $X \in \mathbf{C o n w}_{C}$ is absolutely $C$-closed and continuous images of absolutely $C$-closed spaces are $C$-Conway, then $C^{\infty}$ is stable.

Consequently, if $C$ is idempotent and continuous images of absolutely $C$-closed spaces are $C$-Conway, then every $X \in \mathbf{C o n w}_{C}$ is absolutely $C$-closed if and only if $C$ is stable.

Proof

(a) Let $X \in \mathbf{C o n w}_{C}$. Assume $X$ is a subspace of $Y \in \mathbf{T y c h}$. It suffices to prove that $X$ is $C$-closed in $\beta Y$. By the stability of $C$ one has $c_{\beta Y}(X)=f\left(c_{\beta X}(X)\right)$, where $f=\beta i$ and $i: X \hookrightarrow Y$ is the inclusion. By $X \in \mathbf{C o n w}_{C}$ we have $X=c_{\beta X}(X)$, so $c_{\beta Y}(X)=f(X)=X$. So $X$ is $C$-closed in $\beta Y$.

(b) Let $M \subseteq X \in \mathbf{C o m p}$. Then $c_{\beta M}^{\infty}(M)$ is $C$-closed in $\beta M$, hence $c_{\beta M}^{\infty}(M) \in \mathbf{C o n w}_{C}$. Let $f=\beta i: \beta M \rightarrow X$, where $i: M \hookrightarrow X$ is the inclusion. Then $f\left(c_{\beta M}^{\infty}(M)\right)$ is $C$-Conway by our hypothesis. By the other hypothesis of ours $f\left(c_{\beta M}^{\infty}(M)\right)$ is absolutely $C$-closed, so must be $C$-closed in $X$. Since $M \subseteq f\left(c_{\beta M}^{\infty}(M)\right) \subseteq$ $c_{X}^{\infty}(M)$, we conclude that $f\left(c_{\beta M}^{\infty}(M)\right)=c_{X}^{\infty}(M)$. This proves that $C^{\infty}$ is stable.

\section{Remark 4.7}

- It follows from (a) of the above proposition that no power $C=S e q^{\alpha}$ is stable (as the $C$-Conway spaces in this case are the Conway spaces, that need not be countably compact, i.e., absolutely $C$-closed).

- We shall see later that for a stable closure operator $C$ continuous images of absolutely $C$-closed spaces need not be $C$-Conway spaces (see Theorem 4.16).

We still do not know whether the closure operators $C$ such that $\mathbf{C o n w}_{C}$ coincides with the class of all absolutely $C$-closed are necessarily stable. This leaves open the question to characterize the closure operators $C$ such that $\mathbf{C o n w}_{C}$ coincides with the class of all absolutely $C$-closed space (see Problem 7.5).

Example 4.8 Since $S e q$ is finer than $C=\Omega, \mathrm{k}, f b$ every $C$-Conway space is also a Conway space, but the converse need not be true. Indeed, discrete spaces are Conway spaces, but they are not $C$-Conway for $C=\Omega, \mathrm{k}, f b$ (take the discrete space $\mathbb{N}$, it is $C$-dense in $\beta \mathbb{N}$, hence cannot be $C$-Conway).

Similarly, a countably compact space need not be $C$-Conway. For an example take any $p \in \beta \mathbb{N} \backslash \mathbb{N}$. Then $X=\beta \mathbb{N} \backslash\{p\}$ is $C$-dense in $\beta \mathbb{N}$, so $X$ is not $C$-Conway (see also Example 4.3). Let us see now that it is countably compact. Let $X=\bigcup_{n} U_{n}$, where $U_{n}$ are open subsets of $X$ (they are open in $\beta \mathbb{N}$ as well, as $X$ is open in $\beta \mathbb{N})$. Let $A_{n}=U_{n} \cap \mathbb{N}$. By density of $\mathbb{N}$ in $\beta \mathbb{N}$, one has $\overline{A_{n}} \supseteq U_{n}$. In case $A_{n}$ is not cofinite in $\mathbb{N}$, the complement $F_{n}$ of $U_{n}$ in $\beta \mathbb{N}$ will be a neighbourhood of $p$. Since $p$ cannot be a a $G_{\delta}$ set (otherwise the compactness of $\beta \mathbb{N}$ would imply that $\beta \mathbb{N}$ has countable character at $p$ contrary to Fact 1.1) and since $\bigcap_{n} F_{n}=\{p\}$, this means that only finitely many $A_{n}$ can eventually be non-cofinite. So for some $n_{0} \in \mathbb{N}$ all $A_{n}$ with $n>n_{0}$ are cofinite. Thus the respective $U_{n}$ are clopen sets of $\beta \mathbb{N}$ so this open cover has a finite subcover. 
Example 4.9 Now we consider an idempotent closure operator $\alpha$ that is not comparable with $\operatorname{Seq}$. For a space $X$ and a subset $M$ of $X$ let

$$
\alpha_{X}(M)=\bigcup\left\{\overline{C_{x} \cap M}: x \in M\right\}
$$

where $C_{x}$ denotes the connected component of $x$ in $X$. Clearly, $\alpha \leq K$, while $\alpha \leq_{h} K$ fails to be true (take a non-closed $M \subseteq X \in \mathbf{T y c h}$, such that $X$ is connected and $\bar{M}$ is totally disconnected). If $X$ is strongly zero-dimensional, then also $\beta X$ is zerodimensional, so $\alpha_{\beta X}=d_{\beta X}$. Therefore $X$ is $\alpha$-closed in $\beta X$ and so $X$ is $\alpha$-Conway. If $X$ is connected, then certainly $X$ is $\alpha$-Conway if and only if $X$ is compact, as $\alpha_{\beta X}=k_{\beta X}$.

\subsection{The Galois Connection Related to Conway's Property}

The above results suggest the following question: Is there a closure operator $C$ such that Dieu $=\mathbf{C o n w}_{C}$ (i.e., the Dieudonné complete spaces are precisely the $C$ Conway spaces) ? In the next example we provide such a closure operator $C$.

Example 4.10 Define the closure operator $C$ as follows: for $M \subset X$ the set $c_{X}(M)$ consists of all $y \in X$ such that there is a net $\left(m_{j}\right)$ in $M$ which converges to $y$ and which is $d$-Cauchy for every continuous pseudometric $d$ of $M$ (i.e., $\left(m_{j}\right)$ is Cauchy in the fine uniformity of $M$ ). Since continuous images of such nets have the same property, one can easily check that this defines indeed a closure operator. The equality $\mathbf{C o n w}_{C}=$ Dieu follows directly from the definitions.

More generally, one may be interested in characterizing the bireflective subcategories of Tych that can be presented as categories of $C$-Conway spaces for appropriate $C$. The following theorem offers a surprisingly simple answer to this problems by showing that every bireflective subcategory $\mathbf{A}$ of Tych has the form $\mathbf{A}=\mathbf{C o n w}_{C_{\mathbf{A}}}$ for appropriate closure operator $C_{\mathbf{A}}$. Moreover, for the same $C, \mathbf{A}$ coincides also with the class of all absolutely $C$-closed spaces. These closure operators will have also two important additional properties.

Theorem 4.11 For every bireflective subcategory $\mathbf{A}$ of Tych there exists a stable $\beta$ hereditary closure operator $C_{\mathbf{A}} \leq_{h} K$ such that $\mathbf{A}=\mathbf{C o n w}_{C_{\mathbf{A}}}$. More precisely, for $M \subseteq$ $X \in$ Tych one has

(1) $M$ is $C_{\mathbf{A}}$-closed in $X$ if $M \in \mathbf{A}$,

(2) $M$ is $c_{\mathbf{A}}$-dense in $X$ if $X=\rho M \in \mathbf{A}$.

Moreover, for every $X \in \mathbf{T y c h}$ the following are equivalent:

(a) $X \in \mathbf{A}$,

(b) $X$ is absolutely $c_{\mathbf{A}}$-closed;

(c) $X \in \operatorname{Conw}_{C_{\mathbf{A}}}$.

Proof Let $X \in \mathbf{T y c h}$, let $M$ be a dense subspace of $X$ and let $i: M \hookrightarrow X$ be the inclusion maps. Then consider the reflection maps $\iota_{X}: X \rightarrow \rho X$ and $\iota_{M}: M \rightarrow \rho M$ 
and the natural map $f=\rho i: \rho M \rightarrow \rho X$. Let $c_{X}(M)$ be the pullback $c_{X}(M)=$ $\iota_{X}^{-1}(f(\rho M))$ :

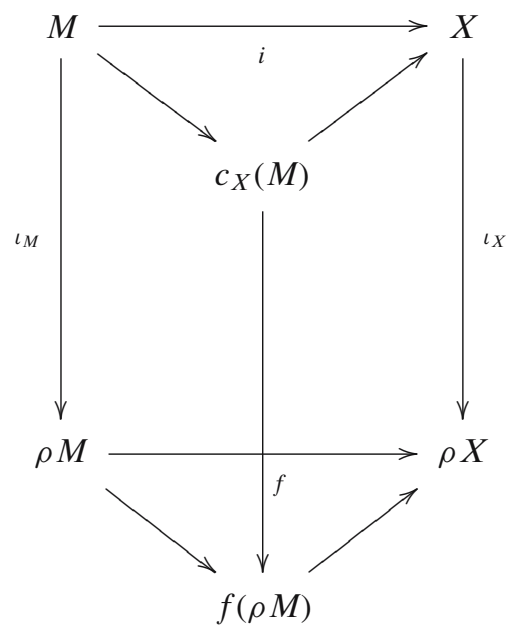

Since $\iota_{X}$ and $f(\rho M) \rightarrow \rho X$ are embeddings, we can write also $c_{X}(M)=f(\rho M) \cap$ $X$. In the case of a dense subspace $M$ the definition of $c_{X}(M)$ coincides with the one given in [9, Definition 3.5] with respect to the discrete closure operator of $\mathbf{A}$ (see also the initial closure operator in $[12,5.13(*)]$ or the pullback closure introduced by Holgate [20,21]). Nevertheless, for the sake of completeness we shall carry out most of necessary steps in the verification that this gives rise to a closure operator. In the general case, let $c_{X}(M)=c_{\bar{M}}(M)$, where $\bar{M}$ is the closure of $M$ in $X$.

Therefore, $X \in \mathbf{C o n w}_{C_{\mathbf{A}}}$ (i.e., $X$ is $C$-closed in $\rho X$ ) if and only if $X=\rho X \in \mathbf{A}$.

Let us show now that the collection $C_{\mathbf{A}}=\left(c_{X}\right)$ defined in this way is a closure operator. To establish monotonicity take $M \subseteq N \subseteq X$ and note that it suffices to consider the case when $M$ is dense in $N$ (otherwise replace $N$ by the closure of $M$ in $N$ ). Next one reduces to the case when $M$ (and so also $N$ ) is dense in $X$. Now an easy diagram chase shows that $c_{X}(M) \subseteq c_{X}(N)$.

Next we need to prove the third property of closure operators. In other words, if $f: X \rightarrow Y$ is a map and $M$ is a subset of $X$, then $f\left(c_{X}(M)\right) \subseteq c_{Y}(f(M))$. Since $f(\bar{M}) \subseteq \overline{f(M)}$, we can assume without loss of generality that $M$ is dense in $X$ and $f(M)$ is dense in $Y$. As in this case the definition coincides with the one given in [12, $5.13(*)]$, we refer the reader to the detailed proof given in [12, Lemma 5.13].

Let us check first (1) and (2). If if $M=\rho M \in \mathbf{A}$, then obviously $c_{\rho M}(M)=M$, so $M$ is $C$-closed. On the other hand, if one has $X=\rho M$, then $\rho X=X$, so that $c_{\rho M}(M)=\rho M$ holds, i.e., $M$ is $C_{\mathbf{A}}$-dense in $X$.

Let us note now that (1) yields the implication (a) $\rightarrow$ (b), while (b) $\rightarrow$ (c) is trivially true for every closure operator. The implication (c) $\rightarrow$ (a) follows from (2) applied to the inclusion $X \hookrightarrow \rho X$ (as $X$ being $C_{\mathbf{A}}$-dense and $C_{\mathbf{A}}$-closed in $\rho X$ implies $X=$ $\rho X)$.

It remains to note, that the equivalence of (a) and (c) is nothing else but the final aim of this proof, namely the equality $\mathbf{A}=\mathbf{C o n w}_{C_{\mathbf{A}}}$.

Finally, let us show that $C=C_{\mathbf{A}}$ is stable and $\beta$-hereditary. To this end it suffices to note that for a dense subset $M$ of an $X \in \mathbf{T y c h}$ one has $\rho M=c_{\beta M}(M)$ and $c_{\beta X}(M)=$ $f(\rho M)$, so $C$ is stable. Moreover, $c_{X}(M)=c_{\beta X}(M) \cap X$ so $C$ is also $\beta$-hereditary. 
The following immediate consequence of Theorem 4.11 is of independent interest since there seems to be no description via closure operators of the bireflective subcategories of Tych.

\section{Corollary 4.12 The correspondences}

$$
C \mapsto \mathbf{C o n w}_{C}, \quad \mathbf{A} \mapsto C_{\mathbf{A}}
$$

are order-reversing. When restricted to closure operators of the form $C_{\mathbf{A}}$ they give rise to a Galois equivalence between the closure operators of $\mathbf{T y c h}$ of the form $C_{\mathbf{A}}$ and bireflective subcategories of $\mathbf{T y c h}$.

Proof The monotonicity shall be established in a more general situation in the next section (see Proposition 5.3). The second assertion follows obviously from Theorem 4.11.

Our next aim is to describe the Galois-closure $G(C)=C_{\mathbf{C o n w}_{C}}$ of a closure operator $C$ in terms of $C$. To this end we define a modification $\widetilde{C}$ of the closure operator $C$ following the pattern used in the proof of Theorem 4.11 for the definition of $C_{\mathbf{A}}$ : for a dense $M \subseteq X \in \mathbf{T y c h}$ let $\widetilde{c}_{X}=X \cap \beta i\left(c_{\beta M}(M)\right)$, where $i: M \hookrightarrow X$ is the inclusion map.

Proposition 4.13 Let $C \leq K$ be a closure operator of Tych.

(a) $\widetilde{C}$ is a stable $\beta$-hereditary closure operator of Tych finer than $\beta(C)$ with $C \leq_{h} K$.

(b) $C$ is stable if and only if $\widetilde{C}=\beta(C)$, consequently $C$ is stable and $\beta$-hereditary if and only if $\widetilde{C}=C$.

(c) $G(C)=G\left(C^{\infty}\right)=\widetilde{C^{\infty}}$.

Proof

(a) To prove that $\widetilde{C}$ is a stable $\beta$-hereditary closure operator it suffices to mimic the proof of Theorem 4.11. The inequality of the second assertion is obvious.

(b) Follows immediately from the definitions.

(c) It suffices to note that $\rho M=c_{\beta M}^{\infty}(M)$ for $\mathbf{A}=\mathbf{C o n w}_{C}$, so (a) applies along with the definition of $C_{\mathbf{A}}$.

Corollary $4.14 G(C) \leq \beta\left(C^{\infty}\right)$ for every closure operator $C \leq K$ of Tych. In particular:

(a) $G(C)=\widetilde{C}$ if $C$ is an idempotent closure operator $C \leq K$ of $\mathbf{T y c h}$, so $G(C) \leq C$ if $C$ is also $\beta$-hereditary.

(b) $G(C)=\beta(C)$ (so $G(C)$ is idempotent) for every idempotent stable closure operator $C \leq_{h} K$ of $\mathbf{T y c h}$.

(c) $G(C)=C$ for every idempotent stable $\beta$-hereditary closure operator $C \leq K$ of Tych. 
Proof $G(C) \leq \beta\left(C^{\infty}\right)$ follows from Proposition 4.13.

(a) The first statement obviously follows from Proposition 4.13. If $C$ is also $\beta$ hereditary, then $C=\beta(C)$ is coarser than $G(C)$ by Proposition 4.13.

(b) follows directly from (b) of Proposition 4.13.

(c) follows from (a) and (b).

We do not know if the closure operators of $\mathbf{T y c h}$ of the form $C_{\mathbf{A}}$ are always idempotent, this is why we say that a bireflective subcategory $\mathbf{A}$ of $\mathbf{T y c h}$ is idempotent if the closure operator $C_{\mathbf{A}}$ is idempotent.

Corollary 4.15 The restriction of the correspondence (4) to the idempotent $\beta$ hereditary closure operators $C \leq_{h} K$ of Tych defines a Galois correspondence between idempotent $\beta$-hereditary closure operators $C \leq_{h} K$ of Tych and idempotent bireflective subcategoriesof Tych. It becomes a Galois equivalence when further restricted to stable idempotent $\beta$-hereditary closure operators $C \leq_{h} \mathrm{~K}$.

Proof To prove the first assertion we have to show that $C_{\mathbf{A}} \leq C$ for a bireflective subcategory $\mathbf{A}$ of Tych and a closure operator $C$ if and only if $\mathbf{A} \supseteq \mathbf{C o n w}_{C}$. The "only if" part follows from monotonicity of $\mathbf{C o n w}_{-}$. If $\mathbf{A} \supseteq \mathbf{C o n w}_{C}$, then the monotonicity of $C_{-}$implies

$$
C_{\mathbf{A}} \leq G(C) .
$$

Moreover, by Corollary 4.14 (a) $G(C) \leq C$ as $C$ is idempotent and $\beta$-hereditary. Along with (5) this yields $C_{\mathbf{A}} \leq C$.

The second assertion follows immediately from the first one and Corollary 4.14 (c).

According to Theorem 4.11 the class $\mathbf{C o n w}_{C}$ coincides with the class of all absolutely $C^{\prime}$-closed spaces for some closure operator $C^{\prime}$ (namely, $C^{\prime}=G(C)=\widetilde{C^{\infty}}$ ). In the next corollary we show that one can take as $C$ precisely $\widetilde{C}$.

Corollary 4.16 Let $C \leq K$ be a closure operator of Tych. Then

$$
\operatorname{Conw}_{C}=\operatorname{Conw}_{C^{\infty}}=\operatorname{Conw}_{\widetilde{C}}=\operatorname{Conw}_{\widetilde{C}^{\infty}}=\operatorname{Conw}_{\widetilde{C}^{\infty}} .
$$

For every $X \in \mathbf{T y c h}$ the following are equivalent:

(a) $X \in \mathbf{C o n w}_{C}$;

(b) $X$ is absolutely $\widetilde{C}$-closed.

(c) $X$ is absolutely $\widetilde{C^{\infty}}$-closed.

Proof Since $c_{\beta X}(X)=\widetilde{c}_{\beta X}(X)$ for every $X \in$ Tych and the same holds true for $C^{\infty}$, we immediately get Eq. 6.

As (a) is equivalent to $X \in \mathbf{C o n w}_{\widetilde{C}}=\mathbf{C o n w}_{\widetilde{C}^{\infty}}$, the equivalence of (a), (b) and (c) follows from Proposition 2.9 and the stability of $\widetilde{C}$ and $\widetilde{C^{\infty}}$.

In particular, the above corollary yields that Conway spaces coincide with the absolutely $\widetilde{S e q}$-closed spaces. We do not have a handy description of $G(C)$ in the 
general case since the definition of $\widetilde{C}$ makes recourse to the Stone-Čech compactification. In the next example we compute explicitly $\widetilde{S e q}$.

Example 4.17 For a space $X \in \mathbf{T y c h}$ and subset $M$ of $X$

$$
\begin{aligned}
\widetilde{s e q_{X}}(M)= & \left\{x \in \operatorname{seq}_{X}(M): \text { such that } x=\lim x_{n}\right. \\
& \text { and } \left.\left(x_{n}\right) \text { is a } C^{*} \text {-Cauchy sequence of } M\right\} .
\end{aligned}
$$

Indeed, assume $y=\lim x_{n}$, where $\left(x_{n}\right)$ is a $C^{*}$-Cauchy sequence of $M$. Then $\left(x_{n}\right)$ must be converging in $\beta M$. Let $x$ be its limit in $\beta M$. Then $f(x)=\lim f\left(x_{n}\right)=\lim x_{n}=y$ where $f=\beta i: \beta M \rightarrow \beta X$ and $i: M \hookrightarrow X$ is the inclusion. The uniquencess of limits gives $y=f(x) \in f\left(\widetilde{s e q}_{\beta M}(M)\right)$. So $y \in \widetilde{s e q_{X}}(M)$.

Conversely, if $y \in \tilde{\operatorname{seq}}{ }_{X}(M)$, then $y=f(x)$ for some $x \in \operatorname{seq} q_{\beta}(M)$. If $x=\lim x_{n}$ for some sequence $\left(x_{n}\right)$ in $M$, then obviously $\left(x_{n}\right)$ is a $C^{*}$-Cauchy sequence of $M$. Moreover, $\lim x_{n}=\lim f\left(x_{n}\right)=y$ in $X$ too.

By Proposition 4.14 Conw $=\mathbf{C o n w}_{S e q}=\mathbf{C o n w}_{S e q^{\infty}}=\mathbf{C o n w}_{\widetilde{S e q}}=\mathbf{C o n w}_{\widetilde{S e q}}{ }^{\infty}$ and the closure operator $G(\operatorname{Seq})=G\left(S e q^{\infty}\right)=\widetilde{S e q^{\infty}}$ corresponding to the category Conw must satisfy $G(\operatorname{Seq}) \leq \beta\left(S e q^{\infty}\right)$. The inequality is proper since the closure operator $\beta\left(S e q^{\infty}\right)$ cannot be stable (as $S e q^{\infty}$ is not stable). On one hand, seemingly $\widetilde{\mathrm{Seq}}^{\infty}$ is not $\beta$-hereditary, so $\widetilde{\mathrm{Seq}}^{\infty}<\beta\left(\widetilde{\mathrm{Seq}}^{\infty}\right)$. The ground for this belief is the fact that no power of a non-idempotent closure operator can be hereditary ([13, Theorem 3.1]). On the other hand, in contrast to $\widetilde{S e q}, C=\widetilde{S e q}{ }^{\infty}$ may fail to be stable. Since $C$ is idempotent, $\beta(C)$ is idempotent too; moreover it is stable iff and only if $C$ is stable. Since $\beta(C)$ is obviously $\beta$-hereditary, it turns out that $C$ is stable if and only if $\beta(C)=G(C)=G($ Seq) (cf. Problem 7.7).

We have no complete intrinsic description of the closure operators of the form $C_{\mathbf{A}}$ (see Question 7.6). In the next example we give an explicit description of $C_{\mathbf{A}}$ in certain cases and show that some specific closure operators cannot be of this form.

\section{Example 4.18}

(a) Obviously, $C_{\text {Comp }}=K$ and $C_{\text {Tych }}=D$.

(b) Let $\lambda$ be an infinite cardinal. Then for the category $\mathbf{B}_{\lambda}$ of all $\lambda$-bounded spaces in Tych the closure operator $C_{\mathbf{B}_{\lambda}}$ coincides with $\Lambda=\left(\lambda_{X}\right)$. Indeed, Proposition 4.14 (c) can be applied to the stable hereditary idempotent closure operator $\Lambda$.

(c) Let us see that $C=s e q^{\infty} \leq K$ is an idempotent closure operator such that $C=C_{\mathbf{A}}$ for no $\mathbf{A}$. Indeed, if such an $\mathbf{A}$ exists, then $C=C_{\mathbf{A}}$ must be stable by Theorem 4.11, while $C=s e q^{\infty} \leq K$ is not stable (see Example 3.6).

(d) The stable closure operators $C=\mathrm{K}$ and $f b$ satisfy $\mathbf{C o n w}_{C}=\mathbf{C o m p}$ and by (a) $C_{\text {Comp }}=K \neq C$. Thus $C$ cannot be of the form $C=C_{\mathbf{A}}$ for any bireflective subcategory of Tych.

Remark 4.19 It is natural to ask whether there exists a closure operator $C$ such that for every $X \in \mathbf{T y c h}$ and every $Y \subseteq X$, the subspace $Y$ is $C$-dense in $X$ precisely when $Y$ is dense and $C^{*}$-embedded in $X$. Let us see now that such a closure operator does not exist. Indeed, if $C$ is such a closure operator then for every $X$ the $C^{*}$-embedding $X \hookrightarrow \beta X$ would entail $X$ is $C$-dense in $\beta X$. Now for every compactification $X \hookrightarrow$ $K$ there exists a continuous map $f: \beta X \rightarrow K$ extending the identity of $X$, so $f$ is 
necessarily surjective. Therefore the $C$-density of $X$ in $\beta X$ implies that $X$ is $C$-dense in $K$ as well by Lemma 3.4 (a). Hence for any compactification $X \hookrightarrow K$ distinct from $\beta X$, the set $X$ is $C$-dense in $K$, but not $C^{*}$-embedded, a contradiction.

\section{Further Generalizations of Conway's Property}

Here we define two generalizations of Conway's property, of which the second generalizes also Conw $_{C}$.

Let us now approach the Conway property in a completely different way, namely: for any bireflective subcategory $\mathbf{A}$ of Tych with bireflection $\rho:$ Tych $\rightarrow \mathbf{A}$ let

$$
\text { Conw }_{\mathbf{A}}=\{X \in \mathbf{T y c h}: X \text { is sequentially closed in } \rho X\}
$$

Note that $\mathbf{C o n w}=\operatorname{Conw}_{\mathbf{C o m p}}=\operatorname{Conw}_{\mathbf{R C o m p}}=\operatorname{Conw}_{\text {Dieu }}$.

Theorem 5.1 Conw is a bireflective subcategory of $\mathbf{T y c h}$ containing A.

Proof Define the bireflection $\rho:$ Tych $\rightarrow \mathbf{C o n w}_{\mathbf{A}}$ by $\rho X=\operatorname{seq}_{\rho X}^{\infty} X$. In other words, $\rho X$ is the smallest sequentially closed subset of $\rho X$ containing $X$. This works.

Each one of the constructions $\mathbf{C o n w}_{C}$ and $\mathbf{C o n w}_{\mathbf{A}}$ depends on a single "parameter" ( $C$ and $\mathbf{A}$, respectively). Now we unify both constructions into a single one depending simultaneously on both "parameters" $C$ and $\mathbf{A}$.

For every closure operator $C$ of Top finer than the Kuratowski closure and a bireflective subcategory $\mathbf{A}$ of $\mathbf{T y c h}$ with reflector $\rho: \mathbf{T y c h} \rightarrow \mathbf{A}$, we can consider the following full subcategory of Tych:

$$
\text { Conw }_{\mathbf{A}, C}=\{X \in \mathbf{T y c h}: X \text { is } C \text {-closed in } \rho X\} .
$$

Clearly, $\operatorname{Conw}_{C}=\operatorname{Conw}_{\mathbf{C o m p}, C}$ and $\mathbf{C o n w}_{\mathbf{A}}=\operatorname{Conw}_{\mathbf{A}, S e q}$. We shall call Conw $\mathbf{C}_{\mathbf{A}}$ the C-Conway hull of $\mathbf{A}$ and we shall prove as before that this gives rise to a bireflective subcategory of $\mathbf{T y c h}$.

\section{Theorem 5.2 Conw $_{\mathbf{A}, C}$ is a bireflective subcategory of $\mathbf{T y c h}$ containing A.}

Proof Define the bireflection $\rho:$ Tych $\rightarrow \mathbf{C o n w}_{\mathbf{A}}$ by $\rho X=c_{\rho X}^{\infty} X$. To check it works one has to mimic the proof of Theorem 3.7 replacing $\mathbf{C o m p}$ by $\mathbf{A}, \beta X$ by $\rho X$ and Seq by $C$.

Proposition 5.3 If $\mathbf{A}_{1}$ and $\mathbf{A}_{2}$ are bireflective subcategories of $\mathbf{T y c h}$ and $C_{1}, C_{2}$ are closure operators of Top. Then $\mathbf{A}_{1} \subseteq \mathbf{A}_{2}$ and $C_{2} \leq C_{1}$ imply

$$
\operatorname{Conw}_{\mathbf{A}_{1}, C_{1}} \subseteq \operatorname{Conw}_{\mathbf{A}_{2}, C_{2}} .
$$

Proof Clearly it is enough to split Eq. 7 in two by proving separately that $\operatorname{Conw}_{\mathbf{A}_{1}, C} \subseteq$ $\operatorname{Conw}_{\mathbf{A}_{2}, C}$ for every closure operator $C$ of $\mathbf{T o p}$, and $\operatorname{Conw}_{\mathbf{A}, C_{1}} \subseteq \operatorname{Conw}_{\mathbf{A}, C_{2}}$ for every bireflective subcategory $\mathbf{A}$ of $\mathbf{T y c h}$. The latter assertions is obvious since $C_{1}$-closed always implies $C_{2}$-closed due to $C_{2} \leq C_{1}$.

To prove the first assertion assume that $X \in \mathbf{C o n w}_{\mathbf{A}_{1}, C}$. In order to check $X \in$ $\operatorname{Conw}_{\mathbf{A}_{2}, C}$ consider the reflection map $\left(\iota_{2}\right)_{X}: X \rightarrow \rho_{2} X \in \mathbf{A}_{2}$. The universal property 
of the map $\left(\iota_{2}\right)_{X}: X \rightarrow \rho_{2} X$ applied to the map $\left(\iota_{1}\right)_{X}: X \rightarrow \rho_{1} X$ gives a map $s: \rho_{2} X \rightarrow \rho_{1} X$ such that

$$
s \circ\left(\iota_{2}\right)_{X}=\left(\iota_{1}\right)_{X} .
$$

Recall that $X$ is $C$-closed in $\rho_{1} X$ by our hypothesis $X \in \mathbf{C o n w}_{\mathbf{A}_{1}, C}$. It follows that the preimage $Z=s^{-1}(X)$ of $X$ under $s: \rho_{2} X \rightarrow \rho_{1} X$ is $C$-closed in $\rho_{2} X$ as a preimage of a $C$-closed set. From Eq. 8 we deduce that $X \subseteq Z$. If $z \in Z$, by the density of $X$ in $Z$ there exists a converging net $x_{\alpha} \rightarrow z$ from $X$. Since $s \uparrow_{X}=i d_{X}$, we get $x_{\alpha}=$ $s\left(x_{\alpha}\right) \rightarrow s(z) \in X$ by Eq. 8. Now the uniqueness of the limit implies $z=s(z) \in X$.

Every closure operator $C$ defines a correspondence Conw $_{-, C}$ that sends every bireflective subcategory A of Tych to its $C$-Conway hull Conw $_{\mathbf{A}, C}$. Since $\mathbf{C o n w}_{-, C}$ can be applied to the latter category as well etc., one may ask whether the iterations of this correspondence can create an ever increasing chain of bireflective subcategories of Tych. The next theorem shows that this chain stabilizes already at the fist step when $C$ is weakly hereditary.

Theorem 5.4 For every bireflective subcategory A of Tych and for every weakly hereditary closure operator $C$ of Top one has

$$
\operatorname{Conw}_{\operatorname{Conw}_{\mathbf{A}, C}, C}=\operatorname{Conw}_{\mathbf{A}, C} .
$$

Proof For the sake of brevity let $\mathbf{B}=\mathbf{C o n w}_{\mathbf{A}, C}$. Assume $X \in \mathbf{C o n w}_{\mathbf{B}, C}$. This means that $X$ is $C$-closed (hence also $C^{\infty}$-closed) in the $\mathbf{B}$-reflection $\rho_{\mathbf{B}} X$. Since $\rho_{\mathbf{B}} X$ was defined precisely as the $C^{\infty}$-closure of $X$ in the $\mathbf{A}$-reflection, it is clear that $X$ is $C^{\infty}$ dense in $\rho_{\mathbf{B}} X$, as $C^{\infty}$ is weakly hereditary whenever $C$ is weakly hereditary [12]. Now $X$ must be simultaneously $C^{\infty}$-closed and $C^{\infty}$-dense in $\rho_{\mathbf{B}} X$, therefore $X=\rho_{\mathbf{B}} X$, so $X \in \mathbf{B}$.

Under the stronger assumption of hereditariness we can establish stability of Conw $_{\mathbf{A}, C}$ under taking $C$-closed subspaces.

Theorem 5.5 For every bireflective subcategory $\mathbf{A}$ of Tych and for every hereditary closure operator $C$ of $\mathbf{T o p}$ the category $\mathbf{C o n w}_{\mathbf{A}, C}$ is stable under taking $C$-closed subspaces.

Proof Assume $X$ belongs to Conw $_{\mathbf{A}, C}$ and let $Y$ be a $C$-closed subspace of $X$. It suffices to see that $Y$ is $C$-closed in its reflection $\rho Y \in \mathbf{A}$. Let $f: \rho Y \rightarrow \rho X$ be the reflection of the inclusion $Y \hookrightarrow X$. Then $f\left(c_{\rho Y}(Y)\right) \subseteq c_{\rho X}(f(Y))=c_{\rho X}(Y)$ by the definition of closure operator. Since $Y=f(Y) \subseteq X=c_{\rho X}(X)$ and the latter is $C$-closed, we conclude that $c_{\rho X}(Y) \subseteq X$. Since $C$ is hereditary, one has $c_{X}(Y)=$ $X \cap c_{\rho X}(Y)=c_{\rho X}(Y)$. Since $Y$ is $C$-closed in $X$ this implies $c_{\rho X}(f(Y))=Y$, so $Y$ is $C$-closed in $\rho X$. Therefore, the third property of closure operators applied to the continuous map $f$ implies

$$
Y=f(Y) \subseteq f\left(c_{\rho Y}(Y)\right) \subseteq c_{\rho X}(Y)=c_{\rho X}(Y)=Y .
$$

This proves that $c_{\rho Y}(Y)=Y$, hence $Y \in \mathfrak{C}_{\mathbf{A}, C}$. 
Applying the above theorem to the hereditary closure operator $C=\operatorname{Seq}$ and $\mathbf{A}=$ Comp we get:

Corollary 5.6 Every sequentially closed subspace of a Conway space is a Conway space.

Analogously, taking $C=\Lambda$ and $\mathbf{A}=\mathbf{C o m p}$, one can deduce that a $\Lambda$-closed subspace of a $\lambda$-bounded space is $\lambda$-bounded, etc.

We end up this section by showing that one can get rid of the forms $\operatorname{Conw}_{\mathbf{A}, C}$ and Conw $_{\mathbf{A}}$ by replacing them with the single form Conw $_{C^{\prime}}$ for an appropriate closure operator $C^{\prime}$ that can be computed in terms of $C$ and $\mathbf{A}$. The price to pay is the more complicated form of the new closure operator $C^{\prime}$. In particular, it may loose some of the nice properties of $C$ that allow for establishing various useful properties of Conw $_{\mathbf{A}, C}$. This should be taken into account in order to realize better the applicability of the next proposition (for the definition of the co-composition $*$ see (*) Section 3.2).

Proposition 5.7 For every bireflective subcategory A of Tych and for every closure operator $C$

$$
\operatorname{Conw}_{\mathbf{A}, C}=\operatorname{Conw}_{C * C_{\mathbf{A}}} \text { and } \operatorname{Conw}_{\mathbf{A}}=\operatorname{Conw}_{S e q * C_{\mathbf{A}}} .
$$

Proof $X \in \mathbf{C o n w}_{\mathbf{A}, C}$ if and only if $X$ is $C$-closed in $\rho X=\left(c_{\mathbf{A}}\right)_{\beta X}(X)$. This means precisely that $X$ is $C * C_{\mathbf{A}}$-closed in $\beta X$, i.e., $X \in \mathbf{C o n w}_{C * C_{\mathbf{A}}}$. The second equality follows from the first one by letting $C=S e q$ and recalling that $\mathbf{C o n w}_{\mathbf{A}, S e q}=\mathbf{C o n w}_{\mathbf{A}}$ by the definition of the latter subcategory.

\section{Conway Property and Topological Groups}

Here we shall give a strong connection of this property to completeness properties of the free topological groups (Section 6.1), as well as a number of further results on topological groups whose underlying space is a Conway space (Section 6.2).

\subsection{Completeness of the Free Topological Groups}

Let TopGr denote the category of Hausdorff topological groups and continuous homomorphisms and let TopAGr be its full subcategory of topological Abelian groups. For a space $X \in$ Tych the free topological group $F(X)$ and the free Abelian topological group $A(X)$ define two functors $F:$ Tych $\rightarrow$ TopGr and $A:$ Tych $\rightarrow$ TopAGr. The following theorem of Graev suggests that the functor $F$ preserves completeness:

Theorem 6.1 (Graev [19]) $F(X)$ is complete, if $X$ is compact.

Indeed, compact spaces are the Tych-closed objects, while in the category TopGr, the TopGr-closed groups (i.e., those that are closed in any other Hausdorff group in which they can be embedded as a topological subgroup) are precisely the complete groups. So $F$ : Tych $\rightarrow$ TopGr sends Tych-closed objects to TopGr-closed ones. 
The above theorem suggests to explore better the preservation and the reflection of closedness (completeness) along the functor $F: \mathbf{T y c h} \rightarrow$ TopGr. Since Dieudonné-complete spaces in Tych can be considered as the complete objects, one can ask:

Problem 6.2 Prove that $F(X)$ (resp. $A(X))$ is complete if and only if the space $X$ is Dieudonné-complete.

This was a long-standing problem in the field of free topological groups. Tkachenko [30] proved that the free Abelian topological group $A(X)$ is complete if and only if the space $X$ is Dieudonné-complete, Uspenskiǐ $[32,33]$ established completeness of $F(X)$ for every metrizable space $X$. A first proof of the general result was given by O. Sipacheva in [28], a final version of the proof appeared only in [29].

One can consider a lower level of completeness for a topological group as follows.

Definition 6.3 A topological group $G$ is sequentially complete, if $G$ is sequentially closed in its Răkov completion.

Now a sequential version of the above problem follows: do the functors $F, A$ : Tych $\rightarrow$ TopGr preserve and reflect sequential completeness [14]? In the category TopGr of topological groups, the sequentially TopGr-closed groups are precisely the sequentially complete groups. According to Theorem 1.9, the countably compact (i.e., absolutely Seq-closed) spaces seem to be the topological counterpart of the sequentially complete groups. It turned out that again, the right context is not that of countably compact spaces, but the larger class of Conway spaces.

The next result (essentially proved in [14]) gives a characterization of the spaces for which the considered free groups are Conway spaces or/and sequentially complete.

Theorem 6.4 For a topological space $X$ the following are equivalent:

(1) $X$ is a Conway space;

(2) $F(X)$ is sequentially complete;

(3) $F(X)$ is a Conway space;

(4) $A(X)$ is sequentially complete.

(5) $A(X)$ is a Conway space.

Proof By Theorem 1.7, if $F(X)$ (resp. $A(X)$ ) is a Conway space, then $X$ is Conway too, being a closed subspace of $F(X)$ (resp. of $A(X)$ ). The remaining implications are either trivial or proved in [14, Theorem 4.13, Theorem 5.1].

It is possible to add in this theorem also the corresponding statements about the free precompact abelian group $P A(X)$ [14, Theorem 5.1].

In [14] this fact is derived from a more precise result that leads to considering the following general problem: Let $X$ be a subspace of $Y$ and suppose that $\mathcal{P}(X, Y)$ is a topological property which describes how $X$ is placed in $Y$. It is natural to ask whether $\mathcal{P}(X, Y)$ always implies $\mathcal{P}(F(X, Y), F(Y))$, where $F(X, Y)$ is the subgroup of $F(Y)$ generated by $X$. A positive answer to this question for sequential closedness was given in [14, Theorem 4.1]. More generally, the topological property $\mathcal{P}(X, Y)$ 
was in all cases of the form: $X$ is $C$-closed in $Y$, where $C$ was one of the closure operators $\mathrm{Seq}, \Omega, \mathrm{K}, \mathrm{fb}$. Hence, in terms of Remark 4.1, one can obtain, in analogy with the above theorem also the following general equivalence: $X \in \mathbf{C o n w}_{C}$ if and only if $F(X) \in \mathbf{C o n w}_{C}$. The next definition allows us to formulate rigorously the theorem below:

Definition 6.5 Call a topological group $G C$-complete if $G$ is $C$-closed in its completion.

Now we can formulate the following theorem essentially proved in [14]:

Theorem 6.6 [14] For $C=S e q, \Omega, k, f b$, and for any $X \in$ Tych the following are equivalent:

(a) $F(X)$ is $C$-complete;

(b) $A(X)$ is $C$-complete;

(c) $X \in \mathbf{C}_{\mathbf{D i}, C}$.

\subsection{Conway Groups}

In this subsection we consider topological groups whose underlying topological space is Conway. In other words, now we are testing the forgetful functor $U:$ TopGr $\rightarrow$ Tych for preservation of sequential completeness. The results will be announced without proof, proofs will appear in [10], where similar bridge between Conway spaces and topological vector spaces will be considered.

For topological groups a stable source for the Conway property is given by the following theorem that can be easily derived from Theorem 1.6.

Theorem 6.7 [10] Every sequentially complete topological group is a Conway space.

Note that this theorem cannot be inverted because any non-complete metrizable group (like $\mathbb{Q}$ ) is Conway, but not sequentially complete. According to Theorem 6.4 these two properties coincide for free topological groups (see Theorem 6.10 below for another instance to this effect).

Since locally countably compact topological groups and topological groups without non-trivial converging sequences are sequentially complete, the theorem gives:

Corollary 6.8 A topological group $G$ is a Conway space if:

(a) $G$ is locally countably compact, or

(b) G has no non-trivial converging sequences.

The fact that topological groups are involved in the above corollary is important. Indeed, the Tychonoff plank $T$ shows that locally compact topological spaces need not be Conway. On the other hand, there exists a pseudocompact non-Conway space without non-trivial converging sequences ([10]).

A topological group $G$ is precompact if its completion $\widetilde{G}$ is a compact group. The precompact groups form an epireflective subcategory PGrp of TopGrp, the reflection of $G \in$ TopGrp is denoted by $G^{+}$. 
Theorem 6.9 For every LCA group $G$, the group $G^{+}$is sequentially complete, hence a Conway space.

In particular, every uncountable abelian group $G$ admits a non-normal and non-countably compact (even non-pseudocompact) group topology that makes it a Conway space. Indeed, consider $G$ as a discrete group and take $G^{+}$. It is not normal [31], nor pseudocompact [5].

The proof of the following theorem from [10] uses the fact that the completion $\widetilde{G}$ of a pseudocompact group $G$ coincides with $\beta G$ (Comfort and Ross [4]).

Theorem 6.10 A pseudocompact group $G$ is a Conway space iff $G$ is sequentially complete.

\section{Open Questions}

Question 7.1 Is there a topological group $G$ such that its Conway reflection $\operatorname{Con} G:=$ $\operatorname{seq}_{\beta G}^{\infty}(G)$ is not a topological group?

Under the assumption of [CH] Berner [2] gave an example of a non-compact space $X$ for which $\beta X$ is a Fréchet-Urysohn topological space. Since such an $X$ is sequentially dense in $\beta X$, it is not a Conway space. Nevertheless, the following questions remains open

\section{Question 7.2}

(a) Is there a Fréchet-Urysohn topological space in ZFC that is not a Conway space?

(b) Is there a consistent example of a topological group whose underlying topological space is Fréchet-Urysohn and non-Conway?

Its counterpart for sequential spaces is open too:

\section{Question 7.3}

(a) Is there a sequential topological space in $\mathrm{ZFC}$ that is not a Conway space?

(b) Is there a sequential topological group that is not a Conway space?

Question 7.4 Does Conw coincide with the bireflective hull (in Tych) of the class of countably compacts spaces (i.e., is every Conway space a sequentially closed subspace of a product of countably compact spaces)?

Problem 7.5 Characterize the closure operators $C$ finer than $K$ such that $\mathbf{C o n w}_{C}$ coincides with the class of all absolutely $C$-closed space.

A partial solution of Problem 7.5 was given in Proposition 2.9.

Corollary 4.15 gave an intrinsic description of the closure operators of Tych the form $C_{\mathbf{A}}$ for an idempotent $\mathbf{A}$ as all stable $\beta$-hereditary idempotent closure operators of Tych finer than $K$. 
Question 7.6 Are all bireflective subcategories of Tych idempotent, i.e., are all closure operators of $\mathbf{T y c h}$ of the form $C_{\mathbf{A}}$ idempotent?

In case of a positive answer to this question, the above intrinsic description extends to all the closure operators of $\mathbf{T y c h}$ the form $C_{\mathbf{A}}$. It will be nice to find the precise relation of these closure operators to the pullback closure of Holgate [20-22].

Our last problem concerns the closure operator $C_{\mathbf{C o n w}}$ associated to the category of Conway spaces as in Theorem 4.11:

Problem 7.7 Prove or disprove the equality $C_{\mathbf{C o n w}}=\beta\left(\widetilde{\operatorname{Seq}}^{\infty}\right)$.

As mentioned in Section 4.2, this equality is equivalent to the stability of the closure operator $\widetilde{S e q}^{\infty}$.

Acknowledgements It is a pleasure to thank the referee and David Holgate for their helpful comments and suggestions.

\section{References}

1. Adámek, J., Herrlich, H., Strecker, G.: Abstract and concrete categories. The joy of cats. In: Pure and Applied Mathematics (New York). Wiley-Interscience, Wiley, New York (1990)

2. Berner, A.J.: $\beta(X)$ can be Frechet. Proc. Amer. Math. Soc. 80, 367-373 (1980)

3. Berri, M.P., Porter, J.R., Stephenson Jr., R.M.: A survey of minimal topological spaces. In: Franklin, S.P., Frolík, Z., Koutnik, V. (eds.) General Topology and Its Relations to Modern Analysis and Algebra, pp. 93-114. Proceedings of the 1968 Kanpur Topology Conference. Akademia, Prague (1971)

4. Comfort, W.W., Ross, K.A.: Pseudocompactness and uniform continuity in topological groups. Pacific J. Math. 16, 483-496 (1966)

5. Comfort, W.W., Saks, V.: Countably compact groups and finest totally bounded topologies. Pacific J. Math. 49, 33-44 (1973)

6. Conway, J.B.: A Course in Functional Analysis. Graduate Texts in Mathematics, 96, pp. xiv+404. Springer, New York (1985)

7. Conway, J.B.: A Course in Functional Analysis, 2nd edn. Graduate Texts in Mathematics, 96, pp. xvi+399. Springer, New York (1990)

8. Dikranjan, D., Gotchev, I.: Sequentially closed and absolutely closed spaces. Boll. Unione Mat. Ital. B (7) 1(3), 849-860 (1987)

9. Dikranjan, D.: Semiregular closure operators and epimorphisms in topological categories. In: V International Meeting on Topology in Italy (Italian) (Lecce, 1990/Otranto, 1990). Rend. Circ. Mat. Palermo (2) Suppl. 29, 105-160 (1992)

10. Dikranjan, D., Martín-Peinador, E., Tarieladze, V.: On a question of Conway. (preprint)

11. Dikranjan, D., Pelant, J.: Categories of topological spaces with sufficiently many sequentially closed spaces. Cahiers Topologie Géom. Différentielle Catég. 38(4), 277-300 (1997)

12. Dikranjan, D., Tholen, W.: Categorical structure of closure operators with applications to topology, algebra and discrete mathematics. In: Mathematics and its Applications, vol. 346, pp. 358+xviii. Kluwer, Dordrecht-Boston-London (1995)

13. Dikranjan, D., Tholen, W., Watson, S.: Classification of closure operators for categories of topological spaces. In: Categorical structures and their applications, pp. 69-98. World Scientific, River Edge, NJ (2004)

14. Dikranjan, D., Tkačenko, M.: Weak complete free topological groups. Topology Appl. 112, 259-287 (2001)

15. Engelking, R.: General Topology. Revised and Completed Edition, pp. viii+529. Heldermann Velag, Berlin (1989)

16. Flor, P.: Zur Bohr-Konvergenz von Folgen. Math. Scand. 23, 169-170 (1968)

17. Gillman, L., Jerison, M.: Rings of continuous functions. In: Graduate Texts in Mathematics, no. 43, pp. xiii+300. Springer, Berlin Heidelberg, New York (1976) 
18. Gotchev, I.: Sequentially $\mathcal{P}$-closed spaces. Rend. Istit. Mat. Univ. Trieste 20,1-17 (1988)

19. Graev, M.I.: Free topological groups, Izvestiya Akad. Nauk SSSR Ser. Mat. 12, 279-323 (1948). English translat. In: Topology and Topological Algebra, Translation Series 1, vol. 8, pp. 305-364. American Mathematical Society (1962)

20. Holgate, D.: The pullback closure operator and generalisations of perfectness. Appl. Categ. Structures 4(1), 107-120 (1996)

21. Holgate, D.: Pullback closure and strong functorial completions. In: Symposium on Categorical Topology (Rondebosch, 1994), 131-139. Univ. Cape Town, Rondebosch (1999)

22. Holgate, D.: Completion and closure. Cahiers Topologie Géom. Différentielle Catég. 41(2), 101-119 (2000)

23. Kannan, V.: Ordinal invariants in topology. Mem. Amer. Math. Soc. 32(245), v+164 (1981)

24. Kim, J.: Sequentially complete spaces. J. Korean Math. Soc. 9, 39-43 (1972)

25. Margaleff Roig, J., Outerelo Dominguez, E., Pinilla Ferrando, J.L.: Topologia, III. Alhambra, Madrid (1980)

26. Reid, G.A.: On sequential convergence in groups. Math. Z. 102, 227-235 (1967)

27. Rudin, M.E.: A normal space $X$ for which $X \times I$ is not normal. Fund. Math. 73, 179-186 (1971)

28. Sipacheva, O.V.: Zerodimensionality and completeness in free topological groups I, II. Serdica 15, 119-140; 141-154 (1989) (in Russian)

29. Sipacheva, O.V.: Free topological groups of spaces and their subspaces. Topology Appl. 101(3), 181-212 (2000)

30. Tkačenko, M.G.: On the completeness of free Abelian topological groups. Soviet Math. Dokl. 27, 341-345 (1983), Russian original In: Dokl. AN SSSR 269, 299-303 (1983)

31. Trigos-Arrieta, J.: Every uncountable abelian group admits a nonnormal group topology. Proc. Amer. Math. Soc. 122(3), 907-909 (1994)

32. Uspenskiǐ, V.V.: On subgroups of free topological groups. Soviet Math. Dokl. 32, 847-849 (1985). Russian original In: Dokl. AN SSSR 285, 1070-1072 (1985)

33. Uspenskiǐ, V.V.: Free topological groups of metrizable spaces, Math. USSR Izvestya. 37, 657-680 (1991). Russian original In: Izvestya AN SSSR 54, N.6. (1990)

34. Wilansky, A.: Topology for Analysis. Ginn, A Xerox Company, pp. ix+383. The College Division, Waltham (1970) 